\title{
Effect of maternal alcohol consumption on gestational diabetes detection and mother-infant's outcomes in Kinshasa, DR Congo
}

\author{
Tandu-Umba Barthélémy*, Mbangama Muela Andy, Mbungu Mwimba Roger
}

Department of OB-GYN, University Clinics, Kinshasa, Congo.

Email: ${ }^{\text {btanduumba@yahoo.fr }}$

Received 11 August 2011; revised 28 September 2011; accepted 16 October 2011.

\begin{abstract}
Objectives: Since it has been suggested that moderate alcohol drinking would increase insulin sensitivity, which could benefit Gestational Diabetes Mellitus (GDM), the study aimed at evaluating alcohol consumption during pregnancy, and seeing whether this consumption influences GDM detection and maternal/perinatal outcomes. Study design: Women with already known diabetes and those with multiple pregnancy were excluded. All other pregnant women attending antenatal care unit of the university clinics, Kinshasa, DR Congo during the period from 1 March throughout 31 October 2010, were invited at 24-week gestation to enroll in $O$ 'Sullivan blood glucose testing and if eligible in 100-gram oral glucose tolerance test. Alcohol consumption, risk factors for GDM, and general characteristics such as age, parity, gestity, BMI, fat mass were registered. Diagnosed GDM was first treated with diet and exercise, thereafter with Metformin, and if necessary with insulin. For other (normal) women data remained blinded until confinement. Maternal and infant's adverse outcomes such as maternal urinary infection, preeclampsia, cesarean section, intrauterine growth retardation, birth weight $<$ $2500 \mathrm{~g}$, birth weight $\geq 3800 \mathrm{~g}$ (as stated $>$ percentile 90 in our milieu), Apgar score at the first minute $<7$, shoulder dystocia or other birth injury, neonatal hypoglycemia and fetal alcohol syndrome (FAS) were compared and analyzed according to GDM diagnosis as well to alcohol status. Results: Up to 240 pregnant women accepted to enroll into the study. Alcohol consumption concerned $78(32.5 \%)$ of the women, most of them $(61=25.42 \%)$ being heavy consumers. Risk factors for GDM and Physical and blood glucose characteristics were alike ( $p$ not significant) in both consumers and non consumers, except for history of HTA in the family that was significantly more frequent $(p=0.02)$ among drinkers. GDM's prevalence
\end{abstract}

was $9 \%$. No adverse outcome was more prominent in any subgroup, except Apgar score $<7$ at the first minute that was more frequent $(p=0.038)$ among neonates of GDM mothers. No FAS, neither shoulder dystocia nor neonatal hypoglycemia were diagnosed. When alcohol status was considered, Birthweight $\geq$ $3800 \mathrm{~g}$ was found more frequent $(p=0.0284)$ in alcohol consumers than in abstainers. Risk of this outcome was three times higher when history of family hypertension was present (odds ratio 2.694; CI: 0.536 - 13.544). Conclusions: The prevalence of alcohol consumption by pregnant women of our series $(32.5 \%)$ seems not to impact the detection of GDM $(9 \%)$. FAS was not diagnosed. Lack of significant differences in adverse outcomes between GDM and non GDM could be attributed to huge follow-up of GDM women. Influence of alcohol consumption on birth weight mostly in setting of familial history of hypertension remains to be addressed.

Keywords: Pregnancy; Alcohol Consumption; GDM; Mother-Infant's Outcomes

\section{INTRODUCTION}

Alcohol is a potent teratogen in humans and both moderate and high levels of alcohol intake during early pregnancy may result in alterations of growth and morphogenesis in the fetus, including the so called fetal alcohol syndrome (FAS) [1-5]. In neonates this syndrome is known as microcephaly associating characteristic face made of short palpebral fissures, sunken nasal bridge, short nose, flattening of the cheekbones and midface, smoothing and elongation of the ridged area (the philtrum) between the nose and lips, and smooth, thin upper lip.

Previously, it has been suggested that moderate drinking would increase insulin sensitivity [6], but no study questioned it as protective or adverse factor on either detection or survey of Gestational Diabetes Mellitus (GDM) 
which is known as a situation of low insulin sensitivity.

This study aims at three issues: to evaluate the importance of alcohol consumption by pregnant women in our milieu; to determine whether alcohol consumption during pregnancy influences GDM detection; to determine its influences on maternal and infant's outcomes among GDM women.

\section{MATERIALS AND METHODS}

This observational study was approved by the institutional review board of the faculty of medicine, University of Kinshasa. Women with already known diabetes were excluded. Multiple pregnancies were also excluded. All other pregnant women attending antenatal care unit of the university clinics, Kinshasa, DR Congo during the period from 1 March throughout 31 October 2010, were invited to enroll in 50-gram glucose O'Sullivan testing at 24-week gestation (calculated from the last menstrual period and early ultrasound examination data). Venous blood glucose was assayed by use of One Touch Profile Meters (Lifescan, Johnson \& Johnson, High Wycombe, U.K.). Based on this testing GDM was defined as blood glucose $\geq 200 \mathrm{mg} / \mathrm{dL}$. Women with values between 140 $\mathrm{mg} / \mathrm{dL}$ and $199 \mathrm{mg} / \mathrm{dL}(\mathrm{n}=38)$ were encouraged to join a 100-gram oral glucose tolerance test (OGTT).

Alcohol consumption was quoted as light (less than 1 litre but more than $30 \mathrm{cl} /$ day) or heavy (more than 1 1/day) [3-5] and no counseling was initiated for drinkers. Maternal characteristics included age, parity, gestity, body mass index (BMI calculated as [weight $(\mathrm{kg}) /$ height (m)2]), and fat mass according to impedancimetry (with an OMRON BF 300 impedance meter). Risk factors for GDM were also registered: obesity (BMI > 25), familyhistory of diabetes (grandparents, parents, brothers, sisters), or of arterial hypertension (HTA), previous history of polyhydramnios, infant's birth weight $\geq 3800 \mathrm{~g}$ ( $>$ 90th percentile in our milieu), stillbirth and congenital malformation. Diagnosed GDM was first treated with diet and exercise, aiming to achieve a fasting blood glucose lower than $95 \mathrm{mg} / \mathrm{dL}$, thereafter with MetforminR, and if necessary with insulin. For other (normal) women data remained blinded until confinement. Weight of the newborn was measured immediately after birth. Infants were examined, and judged clinically as having or not signs of effect of alcohol on morphogenesis.

\subsection{Maternal and Infant's Adverse Outcomes}

Such as maternal urinary infection, preeclampsia, cesarean section, intrauterine growth retardation (IUGR), birth weight $\geq 3800 \mathrm{~g}$, Apgar score $<7$ at the first minute, shoulder dystocia or other birth injury, clinical neonatal hypoglycemia and FAS were compared according to both GDM and alcohol status.

\subsection{Statistical Analysis $(p<0.05$ Significant)}

Differences between means were calculated using Student's $t$ test (for normally distributed results) or otherwise according to Mann-Whitney test. Differences between proportions were calculated according to chisquare or Fischer's exact test where appropriate. Multivariate adjusted odds ratios ( $95 \%$ confidence interval) were used to eliminate influence of variables that could modify effect of alcohol consumption on GDM diagnosis as well as on maternal and infant's adverse outcomes.

\section{RESULTS}

Up to 240 pregnant women accepted to enroll into the study. Their gestational age at delivery ranged from 37 to 41 weeks. Gestational age at recruitment was $30.78 \pm$ 4.6 weeks. The birth weight at term was $3132.97 \pm 470.1$ g. Other characteristics means at recruitment are presented in Table 1.

Table 1. Maternal characteristics according to alcohol status (Mean \pm Standard deviation).

\begin{tabular}{|c|c|c|c|c|}
\hline & Overall group $(n=240)$ & Alcohol abstainers $(n=162)$ & Alcohol drinkers $(n=78)$ & p values \\
\hline Age (yrs) & $31.25 \pm 4.9$ & $30.94 \pm 5.03$ & $31.88 \pm 4.6$ & 0.1699 \\
\hline Parity & $1.99 \pm 1.84$ & $1.93 \pm 1.89$ & $2.1 \pm 1.7$ & 0.5041 \\
\hline Gestity & $3.0 \pm 2.08$ & $2.85 \pm 2.17$ & $3.29 \pm 1.8$ & 0.1244 \\
\hline BMI $\left(\mathrm{kg} / \mathrm{m}^{2}\right)$ & $24.73 \pm 4.4$ & $24.65 \pm 4.3$ & $24.88 \pm 4.6$ & 0.7051 \\
\hline Fat mass $(\mathrm{kg})$ & $19.88 \pm 7.7$ & $19.6 \pm 7.6$ & $20.45 \pm 7.8$ & 0.4326 \\
\hline Fat mass $(\%)$ & $27.5 \pm 6.5$ & $27.5 \pm 6.2$ & $27.8 \pm 7$ & 0.7190 \\
\hline Plasma glucose during O'Sullivan (mg/dL) & $121.10 \pm 30.7$ & $122.2 \pm 31.2$ & $121.9 \pm 30.8$ & 0.4071 \\
\hline GDM after O'Sullivan & 6 & $5(3.1 \%)$ & $1(1.3 \%)$ & 0.7190 \\
\hline GDM & 21 & $13(8 \%)$ & $8(10.2 \%)$ & 0.6071 \\
\hline
\end{tabular}


Alcohol consumption concerned $78(32.5 \%)$ of the women attending our antenatal care unit, most of them ( $61=25.42 \%$ of the overall series) being heavy consumers. Beer was the only type of beverage reported. The most invoked reason for alcohol consumption was the prevention of discomforting nausea and vomiting.

No significant difference was found between alcohol consumers and abstainers. Out of 240 women registered for O'Sullivan testing 6 were recognized GDM ( $\geq 200$ $\mathrm{mg} / \mathrm{dL}$ of blood glucose value) and 188 were O'Sullivan negative (glucose values less than $140 \mathrm{mg} / \mathrm{dL}$ ). Among 46 women who had glucose values ranging from 140 $\mathrm{mg} / \mathrm{dL}$ and $199 \mathrm{mg} / \mathrm{dL} 11$ failed to join OGTT and were excluded for the calculation of GDM's prevalence which thus concerned 6 women diagnosed after O'Sullivan testing and 15 after OGTT $(21 / 229=9 \%)$.

Risk factors for GDM were alike (p not significant) in both alcohol consumers and non consumers, except for familial history of HTA that was more frequent $(p=0.02)$ among drinkers (Table 2).

Eight women who delivered before term or at another maternity were excluded for mother/infant's outcomes assessment, which thus restricted further calculations to 221 mother/infant couples (Tables 3-4).

No adverse outcome was more prominent in any subgroup, except Apgar score $<7$ at the first minute that was more frequent $(p=0.038)$ among neonates of GDM mothers. No FAS, neither shoulder dystocia nor neonatal hypoglycemia were diagnosed. When alcohol status was considered (Table 4), birth weight $\geq 3800 \mathrm{~g}$ was found more frequent in alcohol consumers than in abstainers ( $p$ $=0.0284)$. Since maternal history of HTA in the family was the only risk factor for GDM more frequent ( $p=$ 0.02) among drinkers, its influence on prominent APO (Preeclampsia, Apgar at the first minute $<7$, birth weight $\geq 3800 \mathrm{~g}$ ) according to alcohol consumption was assessed using multivariate adjusted odds ratios ( $95 \%$ confidence interval): odds ratio of 1.067 (CI: $0.129-8.813$ ), 0.975 (CI: 0.057 - 3.061 ) and 2.694 (CI: $0.536-13.544$ ) for Preeclampsia, Apgar 1'<7, and birth weight $\geq 3800$ $\mathrm{g}$ respectively. This means that a history of hypertension in the family multiplies by three the risk of having an infant $\geq 3800 \mathrm{~g}$ in alcohol drinkers.

Table 2. Risk factors for GDM according to alcohol status.

\begin{tabular}{ccccc}
\hline & Overall group $(\mathbf{n}=\mathbf{2 4 0})$ & Abstainers $(\mathbf{n = 1 6 2})$ & Drinkers $(\mathbf{n}=\mathbf{7 8})$ & p values \\
\hline Maternal age $\geq 35$ years & $64(26.7 \%)$ & $42(25.9 \%)$ & $22(34.4 \%)$ & 0.139 \\
History of diabetes in the family & $79(32.9 \%)$ & $51(31.5 \%)$ & $28(35.9 \%)$ & 0.594 \\
History of HTA in the family & $19(7.9 \%)$ & $8(4.9 \%)$ & $11(14 \%)$ & 0.026 \\
History of Macrosomia & $31(12.9 \%)$ & $22(13.6 \%)$ & $9(12 \%)$ & 0.803 \\
History of stillbirth & $15(6.3 \%)$ & $13(8 \%)$ & $2(3 \%)$ & 0.182 \\
History of polyhydramnios & $4(1.7 \%)$ & $3(1.9 \%)$ & $1(1.5 \%)$ & 0.844 \\
History of congenital malformation & 0 & 0 & 0 & 0.406 \\
BMI $>25 \mathrm{~kg} / \mathrm{m}^{2}$ & $91(37.9 \%)$ & $58(35.8 \%)$ & $5(6 \%)$ & \\
\hline
\end{tabular}

Table 3. Mother/infant's adverse outcomes according to GDM status $(\mathrm{N}=221)$.

\begin{tabular}{|c|c|c|c|}
\hline Preeclampsia & $\operatorname{GDM}(\mathbf{n}=\mathbf{2 0}) 2(10 \%)$ & Non GDM $(\mathbf{n}=\mathbf{2 0 1}) 17(8.5 \%)$ & p values 0.814 \\
\hline Cesarean section & $9(45 \%)$ & $55(27.4 \%)$ & 0.097 \\
\hline Apgar score $1{ }^{\prime}<7$ & $3(15 \%)$ & $6(3 \%)$ & 0.038 \\
\hline Birthweight $<2500$ gr & $1(5 \%)$ & $10(4.9 \%)$ & 0.657 \\
\hline Birthweight $\geq 3800$ gr & $2(10 \%)$ & $16(7.9 \%)$ & 0.502 \\
\hline Shoulder dystocia or & 0 & 0 & - \\
\hline \multicolumn{4}{|l|}{ other birth injury } \\
\hline Neonatal hypoglycemia & 0 & 0 & - \\
\hline FAS & 0 & 0 & - \\
\hline
\end{tabular}


Table 4. GDM diagnosis and mother/infant's adverse outcomes according to alcohol status $(\mathrm{N}=221)$.

\begin{tabular}{|c|c|c|c|}
\hline & Alcohol drinkers $(\mathrm{n}=71)$ & Alcohol abstainers $(\mathrm{n}=150)$ & $\mathrm{p}$ values \\
\hline GDM & $7(9.9 \%)$ & $13(8.7 \%)$ & 0.773 \\
\hline Preeclampsia & $6(8.5 \%)$ & $13(8.7 \%)$ & 0.957 \\
\hline Cesarean section & $23(32.4 \%)$ & $41(27.3 \%)$ & 0.439 \\
\hline Apgar score $1^{\prime}<7$ & $4(5.6 \%)$ & $5(3.3 \%)$ & 0.419 \\
\hline Birthweight $<2500 \mathrm{~g}$ & $2(2.8 \%)$ & $9(6 \%)$ & 0.310 \\
\hline Birthweight $\geq 3800 \mathrm{~g}$ & $10(14.1 \%)$ & $8(5.3 \%)$ & 0.0284 \\
\hline
\end{tabular}

\section{COMMENTS}

When compared to the prevalence found five years ago $(5.2 \%)$ in a multicentre study in Kinshasa [7] the rate of this one-hospital-based study (9\%) is much higher, probably due to differences in study population sampling, but a real rise should be questioned. Nevertheless, it is expected to rise with use of lower blood glucose standards recently recommended by the 6th Symposium on Pregnancy \& Diabetes held in Salzburg, Austria, in March 2011, which emphasized the HAPO study [8] and seems to have got global agreement on the "one-step diagnosis" of gestational diabetes mellitus (GDM). This makes GDM range among epidemic problems to be faced in our milieu, not only for pregnancy outcomes but mostly for prevention strategies, since GDM is likely to announce type 2 diabetes.

In respect of alcohol consumption during pregnancy, it is known that South Africa has the highest rate of FAS in the world [9], but data from other African countries are scarce to find. The rate of drinkers among pregnant women of our series $(32.5 \%)$ is much higher than that of $4.4 \%$ reported in an Indian series [10]) and in North America (10\% in USA and $17 \%-25 \%$ in Canada [11]). Rates reported in Europe vary considerably from $34 \%$ in France [12] to $81 \%$ in Ireland [13]. Due to feeling of guilt or shame likely to accompany self-reporting of alcohol consumption by pregnant women [14], the actual rate of our study might have to be higher.

Side effects related to alcohol consumption have been reportedly noticeable even with light drinking, which led to the "no alcohol at all during pregnancy" recommendation $[15,16]$. Since most consumers of our series were heavy ones, many fetuses were expected to be at risk. Literature related to influences of alcohol consumption on pregnancy generally refers to evaluation of consumption during the first half of pregnancy [1-5]. Our study deals with the second half but we have supposed that drinking habits might be similar months before. This supposition is supported by the fact that the most in- voked reason for alcohol consumption was the prevention of discomforting nausea and vomiting.

Lack of FAS in our series could thus be linked to failure to diagnose this condition in newborns, or to a lesser sensibility of fetuses of our milieu, which remains to be addressed. Mullally et al. [13] also observed only 3 cases $(0.005 \%)$ of FAS among women whose up to $81 \%$ were alcohol consumers.

As of GDM related mother/infant's adverse outcomes lack of significant differences between GDM and non GDM women (except for Apgar score $<7$ at the first minute) could be attributed to huge follow-up of GDM women while results remained blinded for others. This finding is consistent with the need to actively treat GDM in order to improve mother-infant's outcomes. Increased risk of Birth weight $\geq 3800 \mathrm{~g}$ by alcohol consumption mostly among women having familial history of HTA is difficult to interpret. Previously, protective effects of moderate drinking on the development of type 2 diabetes in elders has been evidenced mostly when compared with heavy drinkers but not with abstainers [17]. No favorable alcohol effect however has been claimed either on GDM detection or survey or on APO in diabetic patients although it has been suggested that moderate drinking would be associated with increased insulin sensitivity [6].

\section{CONCLUSIONS}

The prevalence of alcohol consumption by pregnant women in our milieu is as high as $32.5 \%$, most of them being heavy drinkers. The prevalence of GDM $(9 \%)$ seems not to be impacted by alcohol consumption. As of mother/infant's adverse outcomes (including FAS) lack of significant differences between GDM and non GDM (except for Apgar score $<7$ at the first minute) is consistent with the need to actively treat GDM. Influence of alcohol consumption on Birth weight $\geq 3800 \mathrm{~g}$ mostly in setting of familial history of HTA remains to be addressed. 


\section{ACKNOWLEDGEMENTS}

We are grateful to colleagues and collaborators from the department of OB-GYN, University Clinics, Kinshasa, DR Congo for invaluable assistance in collecting data and caring for pregnant women.

\section{REFERENCES}

[1] Hanson, J.W., Streissguth, A.P. and Smith, D.W. (1978) The effects of moderate alcohol consumption during pregnancy on fetal growth and morphogenesis. Journal of Pediatrics, 92, 457-460.

doi:10.1016/S0022-3476(78)80449-1

[2] Little, R.E. (1990) Moderate alcohol use during pregnancy and decreased infant birth weight. Pediatrics, 85, $1-9$.

[3] Ebrahim, S.H., Anderson, A.L. and Floyd, R.L. (1999) Alcohol consumption by reproductive-aged women in the USA: An update on assessment, burden and prevention in the 1990s. Prenatal and Neonatal Medicine, 4, 419-430.

[4] Mukherjee, R.A.S., S. Hollins, M.T. and Abou-Saleh, J.T. (2005) Low level alcohol consumption and the fetus. British Medical Journal, 330, 375-376. doi:10.1136/bmj.330.7488.375

[5] Strandberg-Larsen, K., Nielsen, N.R., Grønbaek, M., Andersen, P.K., Olsen, J. and Andersen, A.M. (2008) Binge drinking in pregnancy and risk of fetal death. $\mathrm{Ob}$ stetrics and Gynecology, 111, 602-609. doi:10.1097/AOG.0b013e3181661431

[6] Joosten, M.M., Chiuve, S.E., Mukamal, K.J., Hu, F.B., Hendriks, H.F. and Rimm, E.B. (2011) Changes in alcohol consumption and subsequent risk of type 2 diabetes in men. Diabetes, 60, 74-79. doi:10.2337/db10-1052

[7] Tandu-Umba, N.F.B., Paka M.A., Mbungu, M.R. and Muls, E. (2009) Détermination de la prévalence du diabète gestationnel et des facteurs associés à Kinshasa. Annales Africaines de Médecine, 3, 321-325.

[8] The HAPO Study Cooperative Research Group (2008) Hyperglycemia and Adverse Pregnancy Outcomes. The New England Journal of Medicine, 358, 1991-2002. doi:10.1056/NEJMoa0707943

[9] O’Connor, M.J., Tomlinson, M., LeRoux, I.M., Stewart,
J., Greco, E. and Rotheram-Borus, M.J. (2011) Predictors of alcohol use prior to pregnancy recognition among township women in Cape Town, South Africa. Social Sciences and Medicine, 72, 83-90. doi:10.1016/j.socscimed.2010.09.049

[10] Potukuchi, P.S. and Rao, P.G. (2010) Problem alcohol drinking in rural women of Telangana region, Andhra Pradesh. Indian Journal of Psychiatry, 52, 339-343. doi:10.4103/0019-5545.74309

[11] Centers for Disease Control and Prevention (2004) Alcohol consumption among women who are pregnant or might become pregnant-United States, 2002. Morbidity and Mortality Weekly Reports, 53, 1178-1181.

[12] Chassevent-Pajot, A., Guillou-Landréat, M., Grall-Bronnec, M., Wainstein, L., Philippe, H.J., Lombrail, P. and Vénisse, J.L. (2011) Étude de prévalence des conduites addictives chez les femmes enceintes dans une maternité universitaire. Journal de Gynécologie, Obstétrique et Biologie de la Reproduction, 40, 237-245.

[13] Mullally, A., Cleary, B.J., Barry J., Fahey, T.P. and Murphy, D.J. (2011) Prevalence, predictors and perinatal outcomes of peri-conceptional alcohol exposure-retrospective cohort study in an urban obstetric population in Ireland. BMC Pregnancy Childbirth, 11, 11-27. doi:10.1186/1471-2393-11-27

[14] King, J.C. (1997) Substance abuse in pregnancy: A bigger problem than you think. Postgraduate Medicine, 102, 135-150. doi:10.3810/pgm.1997.09.313

[15] Jacobson, J. and Jacobson, S. (1999) Drinking moderately and pregnancy effects on child development. Alcohol Research and Health, 23, 25-30.

[16] Carson, G., Cox, L.V., Crane, J., Croteau, P., Graves, L., Kluka, S., Koren, G., Martel, M.J., Midmer, D., Nulman, I., Poole, N., Senikas, V. and Wood, R. (2010) Society of obstetricians and gynaecologists of canada. Alcohol use and pregnancy consensus clinical guidelines. Journal of Obstetrics and Gynaecology of Canada, 32, S1-S31.

[17] Liu, C., Yu, Z., Li, H., Wang, J., Sun, L., Qi, Q. and Lin, X. (2010) Associations of alcohol consumption with diabetes mellitus and impaired fasting glycemia among middle-aged and elderly Chinese. BMC Public Health, 10, 713-728. doi:10.1186/1471-2458-10-713 